Research Paper

\title{
Amelioration of estrogen-deficiency-induced obesity by Ocimum gratissimum
}

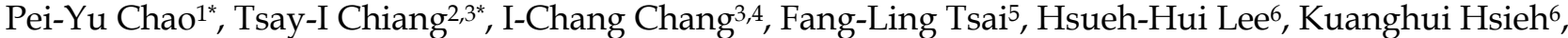 \\ Yung-Wei Chiu'7,8, Te-Jen Lai ${ }^{7,9}$, Jer-Yuh Liu ${ }^{5,10 \bowtie, ~ L i-S u n g ~ H s u ' ~}{ }^{11,12 \bowtie}$, Yang-Chia Shih' ${ }^{13 凶}$ \\ 1. Graduate Institute of Basic Medical Science, China Medical University, Taichung, Taiwan \\ 2. Department of Nursing, College of Medicine \& Nursing, HungKuang University, Taichung, Taiwan \\ 3. School of Medicine, Chung Shan Medical University, Taichung, Taiwan \\ 4. Department of Orthopedic Surgery, Chung Shan Medical University, Taichung, Taiwan \\ 5. Center for Molecular Medicine, China Medical University Hospital, Taichung, Taiwan \\ 6. Department of Laboratory Medicine, Kuang Tien General Hospital, Taichung, Taiwan \\ 7. Institute of Medicine, Chung Shan Medical University, Taichung, Taiwan. \\ 8. Emergency department and center of Hyperbaric Oxygen Therapy, Tungs' Taichung MetroHarbor Hospital, Taichung Taiwan \\ 9. Department of Psychiatry, Chung Shan Medical Hospital, Taichung, Taiwan \\ 10. Graduate Institute of Biomedical Sciences, China Medical University, Taichung Taiwan \\ 11. Institute of Biochemistry, Microbiology and Immunology, Medical College, Chung-Shan Medical University, Taichung, Taiwan \\ 12. Clinical Laboratory, Chung Shan Medical University Hospital, Taichung, Taiwan \\ 13. Department of Biotechnology, Asia University, Taichung, Taiwan \\ *These authors contributed equally to this work.
}

$\square$ Corresponding authors: Jer-Yuh Liu PhD., Graduate Institute of Cancer Biology, College of Medical, China Medical University, No 6, Hsueh-Shih Road, Taichung 40402, Taiwan. Tel: +886-4-22052121 ext 7932; Fax: +886-4-22347028. E-mail: jyl@mail.cmu.edu.tw; Li-Sung Hsu, Ph.D., Institutes of Biochemistry and Biotechnology, Chung Shan Medical University, No. 110, Sec. 1, Jian-Guo N. Road, Taichung 40201, Taiwan. Tel: +886-4-24730022 ext. 11682, Fax: +886-4-23248195, E-mail: 1shsu405@yahoo.com.tw, Yang-Chia Shih: Department of Biotechnology, Asia University, No. 500, Liufeng Rd., Wufeng, Taichung 41354, Taiwan. Tel: +886-4-23323456 ext. 1862, E-mail: angelashih@asia.edu.tw.

(C) Ivyspring International Publisher. This is an open access article distributed under the terms of the Creative Commons Attribution (CC BY-NC) license (https:// creativecommons.org/licenses/by-nc/4.0/). See http://ivyspring.com/terms for full terms and conditions.

Received: 2017.03.05; Accepted: 2017.05.17; Published: 2017.07.20

\begin{abstract}
Objectives: Menopausal transition in women initiates with declining estrogen levels and is followed by significant changes in their physiological characteristics. These changes often lead to medical conditions, such as obesity, which is correlated with chronic low-grade/subclinical inflammation. Ocimum gratissimum L. is a food spice or traditional herb in many countries; the plant is rich in antioxidants, which possess anti-inflammation activities and multitude of other therapeutic functions.

Methods: In this study, we evaluated effects of $O$. gratissimum extract (OGE) in preventing obesity by using ovariectomized (OVX) animal models to mimic menopausal women.

Methods: OVX rats showed increase in body weight and in adipocyte size in perigonadal adipose tissue $(p<0.05)$ and decrease in uterus weight. By contrast, OGE $(0.2 \mathrm{mg} / \mathrm{ml})$ significantly reduced body weight gain and adipocyte in OVX rats and showed insignificant changes in uterus weight. Further investigation indicated that OGE exerted no influence on levels of dorsal fat, serum total cholesterol, and serum triacylglycerol and on serum biochemical factors, calcium, phosphorus, and glucose.

Conclusion: These findings suggested that OGE dietary supplements may be useful in controlling body weight of menopausal women.
\end{abstract}

Key words: Ocimum gratissimum, obesity, ovariectomized animal models

\section{Introduction}

Onset of menopause in females is often associated with increased visceral fat accumulation, a condition known as central obesity [1], and subsequent increase in incidence of hypertension, atherosclerosis, metabolic syndrome, and cardiovascular diseases. Although body composition 
of postmenopausal women results from long-term interactions among between intake, energy expenditure, and sex hormonal status or combination thereof [2], research remains ongoing regarding methods of alleviating menopausal symptoms. Krumm et al. [3] reported that physical activity is an important component of long-term weight maintenance in postmenopausal women. However, in a recent large prospective study among women consuming normal diet, although physical activity was inversely related to weight gain among normal-weight women, no relation was observed among heavy-weight women [4]. Result of such studies invoked interest on other factors that may be involved in preventing physical activities from affecting weight reduction among obese menopausal women.

As inflammation contributes to progression of obesity and its comorbidities [5], numerous studies reported that women undergoing menopausal transition exhibit deleterious changes in circulating inflammatory markers and adipokines correlated with increased visceral adiposity [6], which plays significant pathophysiological role in development of metabolic syndrome and its sequelae, possibly because it serves as source of a number of substances, including free fatty acids and adipokines, such as adiponectin, tumor necrosis factor- $\alpha$, and plasminogen activator inhibitor type 1 [7-9]. These observations highlighted the importance of inflammation to menopause-associated obesity.

Ocimum gratissimum is a commonly used herbal ingredient in Traditional Chinese medicine and widely distributed in tropical and warm-temperate geolocations. O. gratissimum aqueous extract (OGE) consists of abundant antioxidants and possesses many therapeutic functions, including anti-inflammation [10], analgesic and spasmolytic [11], antidiarrheal [12], antitumor [13-17], antiviral [18], and antihyperglycemic activities $[19,20]$. This extract also improves phagocytic functions without affecting humoral or cell-mediated immune system [21]. Our report revealed that expressions of cell cycle regulators, apoptosis-related factors, and cell proliferation markers modified by OGE treatment cause cell apoptosis in human osteosarcoma U2-OS and HOS cells [17]. Antitumor activities of OGE were also observed in breast, prostate, and lung cancers [13-16]. With its many antioxidant components, OGE also exhibits potential to protect body organs from free radical damage and oxidative stress [22-28].

In obesity models, ovariectomy can induce weight gain and fat deposition in rodents, whereas estradiol or antioxidant treatment can restore these changes [29]. Therefore, we investigated effects of
OGE in alleviating obesity-related inflammation by using ovariectomized (OVX) animal models.

\section{Materials and Methods}

\section{Preparation of OGE and composition analysis}

OGE was prepared as described in [22]. $O$. gratissimum leaves were harvested and washed in clear running water and air-dried for 1 week to obtain coarse powders. Powdered plant materials $(20 \mathrm{~g})$ were homogenized with distilled water $(1000 \mathrm{ml})$ using a polytron. Homogenate was boiled for $1 \mathrm{~h}$ and then filtered through two-layer gauze. Filtrate was centrifuged at $20,000 \times \mathrm{g}$ and $4{ }^{\circ} \mathrm{C}$ for $30 \mathrm{~min}$ to remove insoluble pellets. Supernatant was collected, lyophilized, and stored at $-20^{\circ} \mathrm{C}$ until usage. Prior to assays, OGE powder was dissolved at indicated concentration. Total polyphenol and flavonoid contents were determined by Folin-Ciocalteau [30] and Lamaison methods [31], respectively. Polyphenol and flavonoid contents were quantified by comparison with pure standards.

\section{OVX Sprague-Dawley rat model}

All female Sprague-Dawley rats (200 g) were purchased from the National Laboratory Animal Center (Taipei, Taiwan). All procedures involving laboratory animal use were in accordance with guidelines of the Institutional Animal Care and Use Committee of China Medical University (IACUC$\mathrm{CMU}$ ) for the care and use of laboratory animals. All experimental procedures were also approved by the IACUC-CMU.

Twenty-four rats were used. Six rats were given sham operation under Nembutal (pentobarbital sodium; $50 \mathrm{mg} / \mathrm{kg}$ body weight) anesthesia. The remaining rats were OVX and were equally divided into second, third, and fourth groups. For drinking, the first (sham) and second (OVX) groups received sterile water only. The third (OVX + OGE (0.2 $\mathrm{mg} / \mathrm{ml})$ ) and fourth (OVX + OGE $(0.1 \mathrm{mg} / \mathrm{ml}))$ groups received OGE solution diluted with sterile water.

All animals were maintained under regulated 12 $\mathrm{h} / 12 \mathrm{~h}$ light-dark illumination cycles at constant temperature $\left(22 \pm 0.5{ }^{\circ} \mathrm{C}\right)$ and humidity $(45 \%-50 \%)$. Food and drinking water were supplied ad libitum. Body weight was measured at indicated time of the day during experiments. After 12 weeks, animals were sacrificed, and blood and perigonadal adipose tissues were immediately obtained. Dorsal fat and uterus of each rat were dissected and weighed.

\section{Histology of adipose tissues}

Perigonadal adipose tissues were fixed in zinc formaldehyde at $4{ }^{\circ} \mathrm{C}$ overnight prior to their transfer 
and storage in phosphate-buffered saline at $4{ }^{\circ} \mathrm{C}$. Tissues were subsequently embedded in paraffin, sectioned, and stained with hematoxylin and eosin. Digital images were captured with an Olympus DX51 light microscope (Tokyo, Japan).

\section{Measurement of serum biochemical markers}

Blood was coagulated by maintenance at room temperature for 15-20 $\mathrm{min}$ and subsequent centrifugation for $10 \mathrm{~min}$. Serum biochemistry analyzer (AU400, Olympus, Tokyo, Japan) was used to measure calcium ion, glucose, inorganic phosphorus, total cholesterol, and triglyceride.

\section{Statistical analysis}

Data were obtained from six measurements and expressed as means \pm SEM. Statistical comparisons were performed using ANOVA. Student's $t$-test was used in two-group comparisons. $p<0.05$ was considered statistically significant.

\section{Results}

OGE supplementation reduced ovariectomyassociated increase in body weight but not uterine weight

Initial investigations determined effects of OGE uptake on estrogen-deficiency-induced obesity. Increased body weight in OVX rats was significantly higher $(p<0.01)$ than that in sham control rats (Figure 1A). After 12 weeks, body weights of sham and OVX rats measured $313 \pm 8$ and $353 \pm 11 \mathrm{~g}$, respectively. Increased body weight of OVX $+0.2 \mathrm{mg} / \mathrm{ml}$ OGE group was significantly lower than that of OVX group $(p<0.01)$ but not that of OVX $+0.1 \mathrm{mg} / \mathrm{ml}$ OGE group.

Observations also determined changes in uterus weight as response to OGE supplementation. Uterus/body weight ratio in OVX group significantly decreased compared with that of sham group $(p<$ 0.05) (Figure 1B). Uterus/body weight ratio in OVX groups with OGE supplement (OVX $+0.1 \mathrm{mg} / \mathrm{ml}$ OGE and OVX $+0.2 \mathrm{mg} / \mathrm{ml}$ OGE) significantly decreased compared with that of sham group $(p$ $<0.01$ ), but observed change was insignificant compared with that of OVX group.

\section{OGE supplementation decreased OVX-associated increase in adipocyte size}

OGE supplementation attenuated weight gain in OVX rats. Investigation on effects of supplementation on adipocyte properties revealed that adipocytes in perigonadal adipose tissues of OVX rats were clearly enlarged compared with those from sham-operated control rats (Figure 2A). High OGE supplementation $(0.2 \mathrm{mg} / \mathrm{ml})$ reduced enlarged adipocyte size in response to ovariectomy. Changes in dorsal fat mass were also analyzed. Ratio of dorsal fat mass/body weight was insignificantly changed among four experimental groups, though dorsal fat mass in OVX group was higher than that in sham group (Figure 2B).

\section{Effects of OGE on blood biochemical factors}

Blood lipid, total cholesterol, and triacylglycerol levels were determined to investigate whether OGE supplementation can maintain lipid metabolism. As shown in Figure 3 (upper panel), serum total cholesterol level was slightly changed in OVX and OVX groups supplemented with OGE $(p>0.05)$. A similar phenomenon was observed in serum triacylglycerol levels (Figure 3, lower panel). Other blood biochemical factors were also determined. Serum calcium, phosphorus, and glucose showed no change in response to ovariectomy or ovariectomy with OGE supplementation (Figure 4).

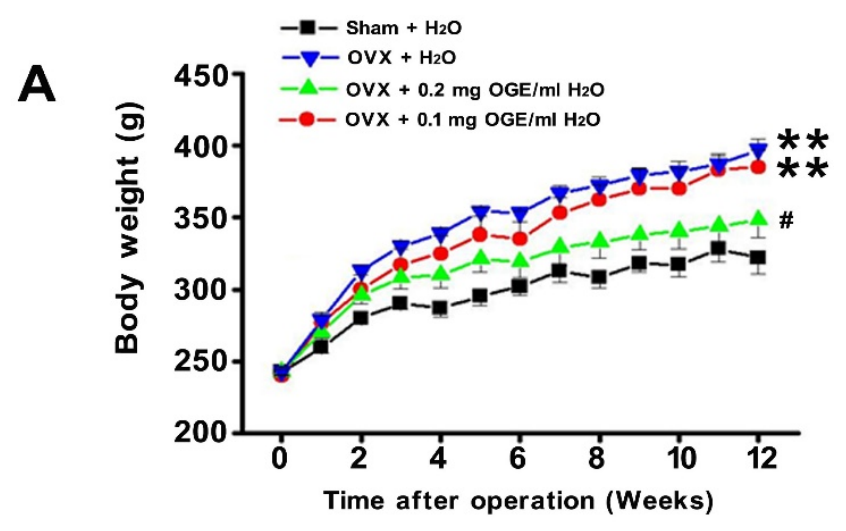

B

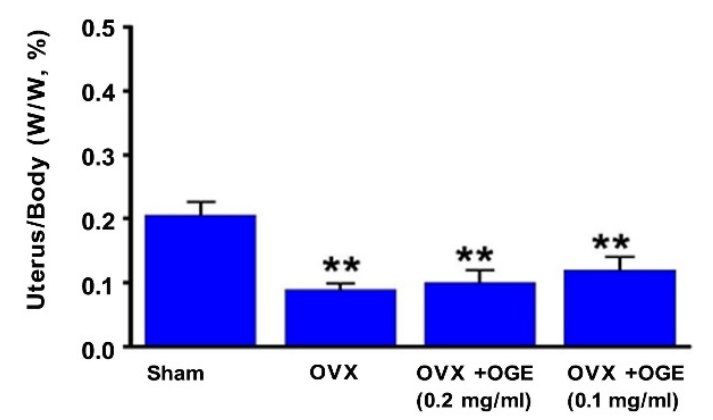

Figure 1. Effects of OGE supplement on body weights. Rats were sham-operated or OVX. OVX rats were divided in three groups; two groups were supplied with water containing OGE $(0.1$ and $0.2 \mathrm{mg} / \mathrm{ml})$, and the other one received sterile water. (A) Body weight was measured each week for 12 weeks. (B) After 12 weeks, rats were sacrificed, and uteruses were obtained and weighed. \#, $p<0.05$ compared with OVX. **, $p<0.01$ compared with sham. 
A

\section{Sham}
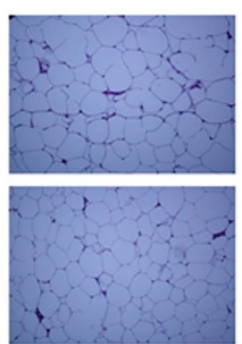

B

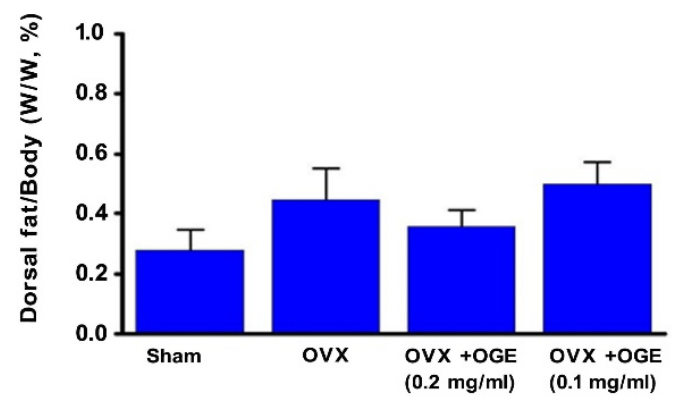

Figure 2. Effects of OGE supplement on adipocyte size and dorsal fat content. Rats were sham-operated or OVX. OVX rats were divided in three groups; two groups were supplied with water containing OGE $(0.1$ and $0.2 \mathrm{mg} / \mathrm{ml})$, and the other one received sterile water. After 12 weeks, rats were weighed and subsequently sacrificed to obtain periuterine adipose tissues and dorsal fat. (A) After hematoxylin and eosin staining, adipocytes were observed by using light microscope (200x). (B) Dorsal fat samples were collected and weighed. n.s.: no statistical significance compared with sham or OVX.
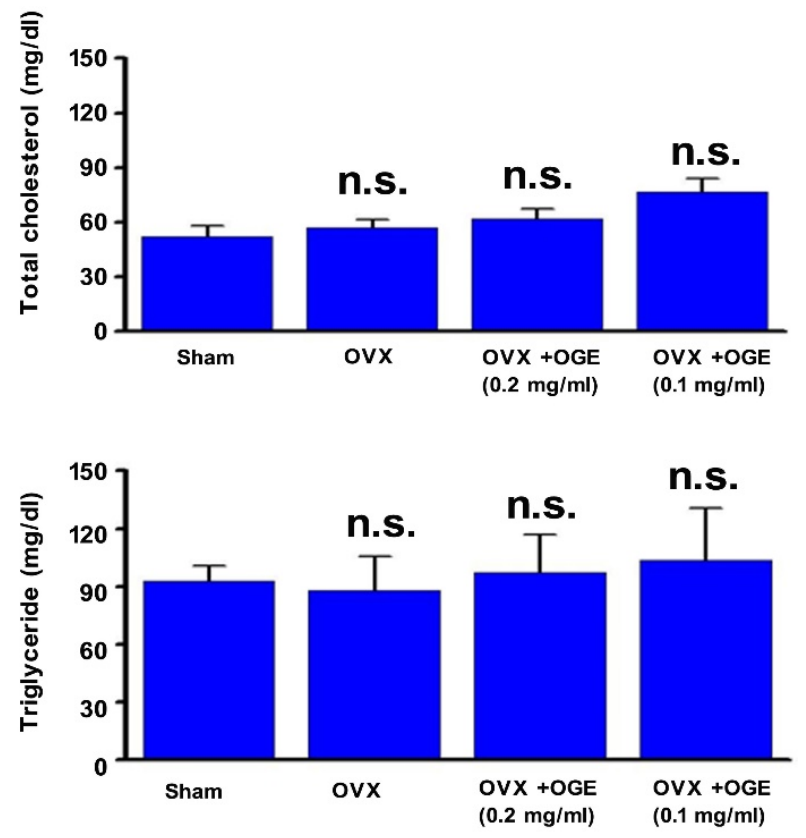

Figure 3. OGE supplement insignificantly affected blood lipids. Rats were sham-operated or OVX. OVX rats were divided in three groups; two groups were supplied with water containing OGE $(0.1$ and $0.2 \mathrm{mg} / \mathrm{ml})$, and the other one received sterile water. After 12 weeks, blood samples were obtained for determination of serum total cholesterol and triacylglycerol. n.s.: no statistical significance compared with sham or OVX.
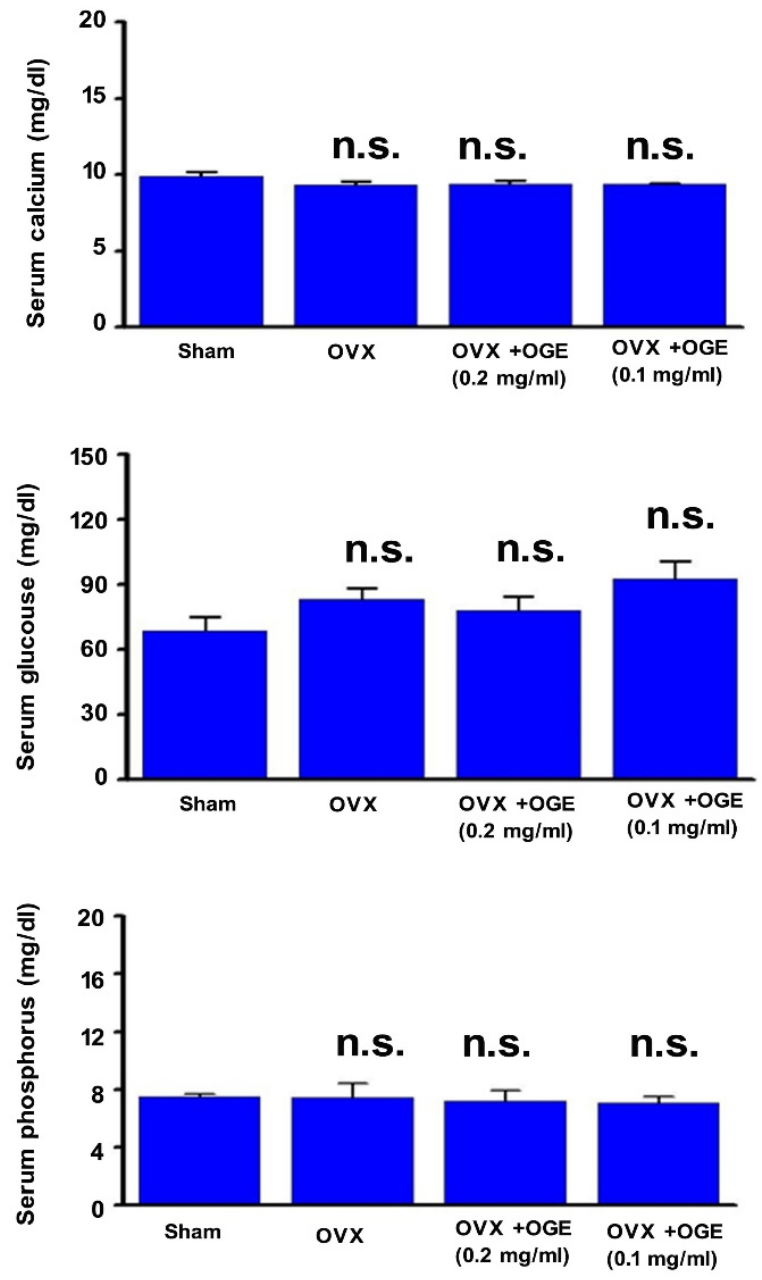

Figure 4. OGE supplement insignificantly affected blood lipids. Rats were sham-operated or OVX. OVX rats were divided in three groups; two groups were supplied with water containing OGE $(0.1$ and $0.2 \mathrm{mg} / \mathrm{ml})$, and the other one received sterile water. After 12 weeks, sera were obtained for determination of calcium, phosphorus, and glucose levels. n.s.: no statistical significance compared with sham or OVX.

\section{Discussion}

Both human (menopause) and rodent (reproductive senescence) studies indicated that agerelated ovarian hormone loss contributes to changes in distribution of adipose tissues [32]. Significant decrease in estrogen and progesterone results from follicular depletion, yielding many androgenic patterns of fat distribution, that is, an increase in central or abdominal adiposity. Similarly, when consuming high-fat diet, male mice and OVX mice can obtain significant increase in body weight [33]. However, treating mice with estrogen results in reduced body weight.

Recently, considerable amount of evidence indicated that flavonoid intake can reduce body fat deposition in OVX animals [34]. Wu et al. [35] demonstrated that combined intervention of flavonoid and exercise prevents body fat accumulat- 
ion in the whole body, increases lean body mass and restoration of bone mass, and reduces high serum cholesterol in OVX mice. These findings suggested that flavonoid intervention can be beneficial by improving both body composition and bone mass in estrogen-deficient condition. As OGE also contains many flavonoids [27], we evaluated effects of OGE in preventing obesity by using the same OVX animal models. The present results show effects of OGE on restoring normal body composition and lipid metabolism under estrogen-deficient conditions, suggesting that OGE supplementation may offer an effective regimen to prevent lifestyle-related health problems.

Reports showed that isoflavone supplementation effectively lowered serum cholesterol in OVX rats [34, 35] and in postmenopausal women [36]. However, other reports showed different results [37]. In the present study, no change was noted in total cholesterol levels in OVX rats. Thus, whether OGE can reverse total cholesterol of OVX rats remains unknown. Possibly, in our previous laboratory, OGE can reduce total cholesterol level in high-fat diet and high-cholesterol diet rats [28].

With significant amount of simple phenols and flavonoids, including catachin and epicatechin [27], discovered in OGE and scientific evidence proving their beneficial anti-obesity effects [38], these compounds may potentially decrease weight gain in OVX rats. Future research should explore molecular mechanisms of such effects. Ovarian adipose tissue macrophages (adipose tissue macrophage clusters) and state of nuclear factor- $\mathrm{kB}$ activation should also be further explored to understand inflammation in estrogen-deficiency-related obesity and natural compounds for clinical use after menopause to achieve weight loss and to improve body figures of menopausal women.

\section{Acknowledgements}

This work was supported by grants from from the Ministry of Science and Technology, Republic of China (MOST 104-2320-B-039-032), as well as in part by Taiwan Ministry of Health and Welfare Clinical Trial and Research Center of Excellence (MOHW106TDU-B-212-113004).

\section{Competing Interests}

The authors have declared that no competing interests exist.

\section{References}

1. Rossi R, Grimaldi T, Origliani G, et al. Menopause and cardiovascular risk. Pathophysiol Haemost Thromb. 2002; 32(5-6): 325-8.
2. Mastorakos G, Valsamakis G, Paltoglou G, et al. Management of obesity in menopause: diet, exercise, pharmacotherapy and bariatric surgery. Maturitas. 2010; 65(3): 219-24.

3. Krumm EM, Dessieux OL, Andrews P et al. The relationship between daily steps and body composition in postmenopausal women. J Womens Health (Larchmt). 2006; 15(2): 202-10.

4. Lee IM, Djoussé L, Sesso HD, et al. Physical activity and weight gain prevention. JAMA. 2010; 303(12): 1173-9.

5. Christensen A, Pike CJ. Menopause, obesity and inflammation: interactive risk factors for Alzheimer's disease. Front Aging Neurosci. 2015; 7: 130.

6. Lee CG, Carr MC, Murdoch SJ, et al. Adipokines, inflammation, and visceral adiposity across the menopausal transition: a prospective study. J Clin Endocrinol Metab. 2009; 94(4): 1104-10.

7. Cnop M, Havel PJ, Utzschneider KM, et al. Relationship of adiponectin to body fat distribution, insulin sensitivity and plasma lipoproteins: evidence for independent roles of age and sex. Diabetologia. 2003; 46(4): 459-69.

8. Katsuki A, Sumida Y, Murashima S, et al. Serum levels of tumor necrosis factor-alpha are increased in obese patients with noninsulin-dependent diabetes mellitus. J Clin Endocrinol Metab. 1998; 83(3): 859-62.

9. Katsuki A, Sumida Y, Murashima S et al. Production of plasminogen activator inhibitor 1 by human adipose tissue: possible link between visceral fat accumulation and vascular disease. J Clin Endocrinol Metab. 1998; 83(3): 859-62.

10. Lin $\mathrm{CC}$, Lin $\mathrm{JK}$, Chang $\mathrm{CH}$. Evaluation of hepatoprotective effects of "Chhit-Chan-Than" from Taiwan. Pharm. Biol. 1995; 33: 139-143.

11. Aziba PI, Bass D, Elegbe Y. Pharmacological investigation of Ocimum gratissimum in rodents. Phytother Res. 1999; 13(5): 427-9.

12. Ilori $\mathrm{M}$, Sheteolu $\mathrm{AO}$, Omonigbehin EA, et al. Antidiarrhoeal activities of Ocimum gratissimum (Lamiaceae). J Diarrhoeal Dis Res. 1996; 14(4): 283-5.

13. Nangia-Makker, P, Tait L, Shekhar MP, et al. Inhibition of breast tumor growth and angiogenesis by a medicinal herb: Ocimum gratissimum. Int $\mathrm{J}$ Cancer. 2007; 121(4): 884-94.

14. Chen HM, Lee MJ, Kuo CY, et al. Ocimum gratissimum aqueous extract induces apoptotic signaling in lung adenocarcinoma cell A549. Evid Based Complement Alternat Med. 2011; 2011: 739093.

15. Nangia-Makker $P, \operatorname{Raz} T$, Tait $L$, et al. Ocimum gratissimum retards breast cancer growth and progression and is a natural inhibitor of matrix metalloproteases. Cancer Biol Ther. 2013; 14(5): 417-27.

16. Ekunwe SI, Hall SM, Luo X, et al. Fractionated Ocimum gratissimum leaf extract inhibit prostate cancer (PC3 $\cdot \mathrm{AR})$ cells growth by reducing androgen receptor and survivin levels. J Health Care Poor Underserved. 2013; 24(4 Suppl): 61-9.

17. Lin CC, Chao PY, Shen CY, et al. Novel Target Genes Responsive to Apoptotic Activity by Ocimum gratissimum in Human Osteosarcoma Cells. Am J Chin Med. 2014; 42(3): 743-67.

18. Ayisi NK, Nyadedzor C. Comparative in vitro effects of AZT and extracts of Ocimum gratissimum, Ficus polita, Clausena anisata, Alchornea cordifolia, and Elaeophorbia drupifera against HIV-1 and HIV-2 infections. Antiviral Res. 2003; 58: 25-33.

19. Aguiyi JC, Obi CI, Gang SS, et al. Hypoglycaemic activity of Ocimum gratissimum in rats. Fitoterapia. 2000; 71(4): 444-6.

20. Casanova LM, da Silva D, Sola-Penna M, et al. Identification of chicoric acid as a hypoglycemic agent from Ocimum gratissimum leaf extract in a biomonitoring in vivo study. Fitoterapia. 2014; 93: 132-41.

21. Atal CK, Sharma ML, Kaul A. et al. Immunomodulating agents of plant origin. I: Preliminary screening. J. Ethnopharmacol. 1986; 18(2): 133-41.

22. Chang HC, Chiu YW, Lin YM, et al. Herbal supplement attenuation of cardiac fibrosis in rats with CCl4-induced liver cirrhosis. Chin J Physiol. 2014; 57(1): 41-7.

23. Li PC, Chiu YW, Lin YM, et al. Herbal Supplement Ameliorates Cardiac Hypertrophy in Rats with CCl4-Induced Liver Cirrhosis. Evid Based Complement Alternat Med. 2012; 2012: 139045.

24. Lee MJ, Chen HM, Tzang BS, et al. Ocimum gratissimum aqueous extract protects $\mathrm{H} 9 \mathrm{c} 2$ myocardiac cells from $\mathrm{H} 2 \mathrm{O} 2$-induced cell apoptosis through Akt signalling. Evid Based Complement Alternat Med. 2011; 2011: 578060.

25. Chiu CC, Huang CY, Chen TY et al. Beneficial Effects of Ocimum gratissimum Aqueous Extract on Rats with CCl4-Induced Acute Liver Injury. Evid Based Complement Alternat Med. 2012; 2012: 736752.

26. Chiu YW, Chao PY, Tsai CC, et al. Ocimum gratissimum is Effective in Prevention against Liver Fibrosis In Vivo and In Vitro. Am J Chin Med. 2014; 42(4): 833-52.

27. Chen YH, Chiu YW, Shyu JC, et al. Protective Effects of Ocimum gratissimum Polyphenol Extract on Carbon Tetrachloride-Induced Liver Fibrosis in Rats. Chin J Physiol. 2015; 58(1): 55-63.

28. Chao PY, Lin JA, Ting WJ, et al. Ocimum gratissmum aqueous extract reduces plasma lipid in hypercholesterol-fed hamsters. Int J Med Sci. 2016; 13(11): 819-24.

29. Wu J, Wang $\mathrm{X}, \mathrm{Chiba} \mathrm{H}$, et al. Combined intervention of soy isoflavone and moderate exercise prevents body fat elevation and bone loss in ovariectomized mice. Metabolism. 2004; 53(7): 942-8.

30. Ainsworth EA, Gillespie KM. Estimation of total phenolic content and other oxidation substrates in plant tissues using Folin-Ciocalteu reagent. Nat Protoc. 2007; 2(4): 875-7.

31. Lamaison JL, Carnat A, Petitjean-Freytet C. [Tannin content and inhibiting activity of elastase in Rosaceae]. Ann Pharm F. 1990; 48(6): 335-40. 
32. Sørensen MB, Rosenfalck AM, Højgaard L, et al. Obesity and sarcopenia after menopause are reversed by sex hormone replacement therapy. Obes Res. 2001; 9(10): 622-6.

33. Stubbins RE, Holcomb VB, Hong $\mathrm{J}$, et al. Estrogen modulates abdominal adiposity and protects female mice from obesity and impaired glucose tolerance. Eur J Nutr. 2012; 51(7): 861-70.

34. Uesugi $\mathrm{T}$, Toda $\mathrm{T}$, Tsuji $\mathrm{K}$, et al. Comparative study on reduction of bone loss and lipid metabolism abnormality in ovariectomized rats by soy isoflavones, daidzin, genistin, and glycitin. Biol Pharm Bull. 2001; 24(4): 368-72.

35. Wu J, Wang $\mathrm{X}, \mathrm{Chiba} \mathrm{H}$, et al. Combined intervention of soy isoflavone and moderate exercise prevents body fat elevation and bone loss in ovariectomized mice. Metabolism. 2004; 53(7): 942-8.

36. Uesugi T, Fukui Y, Yamori Y. Beneficial effects of soybean isoflavone supplementation on bone metabolism and serum lipids in postmenopausal japanese women: a four-week study. J Am Coll Nutr. 2002; 21(2): 97-102.

37. Potter SM, Baum JA, Teng H, et al. Soy protein and isoflavones: their effects on blood lipids and bone density in postmenopausal women. Am J Clin Nutr. 1998; 68(6 Suppl): 1375S-1379S

38. Suzuki T, Pervin M, Goto S. et al. Beneficial Effects of Tea and the Green Tea Catechin Epigallocatechin-3-gallate on Obesity. Molecules. 2016; 21(10): E1305. 